\title{
Organic Garbage Disposal Equipment Management System Based on WSN
}

\author{
Liu Bocheng \\ School of Software, Nanchang University \\ Nanchang, China \\ e-mail: jsjjcjx@163.com \\ Cao Ye \\ School of Software, Nanchang University \\ Nanchang, China \\ e-mail: 13767959389@163.com
}

\author{
Song Jialei \\ School of Software, Nanchang University \\ Nanchang, China \\ e-mail: ncusc_bysj@163.com
}

\begin{abstract}
Huge amount of organic garbage be produced with urbanization rapid development. Garbage disposal equipment were put into use in large scale. Traditional measures of equipment management is no more efficient. This paper focuses on realizing informationization and systematization of equipment management through software technology.
\end{abstract}

\section{Keywords-Garbage Disposal; Equipment Management; Ensor; WSN}

This paper analyzed the requirement of the system and related work process. The system should be able to manage basic information of staff and equipment, receiving data returned from sensors and reminding staff when equipment is abnormal. Staff can set the operation status of equipment via the system. Based on modular programming ideas, then the paper designed the functional modules: personnel management, rights management, equipment management, real-time simulation, task management and fault management. Finally complete the full function of the system, providing the information management way for the management of organic waste equipment, improving the efficiency of the staff and enhancing the modern management level of the enterprise.

\section{INTRODUCTION}

The staff can manage the equipment and tasks and monitor the real-time status of the equipment through computer. Therefore, it can improve the efficiency of equipment management, reduce the enterprise's investment in human resources and promote the informatization of management[1].

The concept of informatization is gradually applied to the management of garbage disposal equipment. According to the analysis of the development trend of China's garbage disposal industry, the waste treatment is gradually adopting high technology and the level is getting higher and higher. In the case of waste separation, the application of automatic equipment, landfill site construction and application of bioengineering technology to improve the efficiency of waste incineration power generation system through thermal physical heat transfer technology[2]. The scale of equipment management is increasing and the complexity of management is improving. The traditional way of equipment management gradually does not meet the demand of production. Network technology can effectively integrate resources and realize sharing, which is being applied to equipment management, thus improving operation and management efficiency[3].

\section{SYSTEM ANALYSIS}

\section{A. System demand analysis}

The main purpose of the system is to improve the management efficiency of waste disposal equipment and realize the network management. The following requirements are summarized by the analysis of the system.

- In order to facilitate user operation, it is necessary to provide a good operation interface. The interface design should conform to the basic aesthetics, the system response speed should be fast, and the operation process should be standardized and reasonable.

- To set different permissions for different personnel roles so as to maintain the system security and run steadily.

- It will be able to manage user information, such as adding, deleting and modifying user information.

- It will be able to manage the basic information of the equipment, such as adding, deleting and modifying basic information of the equipment.

- The operator can carry out task management, add tasks according to the arrangement, and can generate task reports and submit them to the administrator for review.

- The operator can through the system to run the equipment parameters settings, such as equipment internal temperature, the humidity inside the equipment, power equipment, etc., so that the equipment will be in good running condition.

- The operator can check the operation of the system in real time and monitor the equipment. 
- In the case of equipment failure, the system can notify operators in different ways, such as sending text messages, page pop-up prompts, etc.

\section{B. System function analysis}

\section{1) User management}

User can check their personal information and change their passwords. The administrator can manage user information after login, such as adding, deleting, modifying user information and modifying users' permission.

\section{2) Task management}

Administrators distribute tasks by the system, distribute them to executives, and review the submitted tasks. The task performer modifies the status of the task according to the progress of the task, and then submits the task or gives it to the next performer.

\section{3) Equipment management}

The operator can manage the basic information by the system, such as add, delete, modify the basic information of the equipment, and can view the equipment operation manual, at the same time they can intuitively see location of the equipment through the map.

\section{4) Device control}

The operator changes the operating parameters of the equipment, such as internal temperature, internal humidity and machine power, so as to control the operation state of the equipment and improve the operation efficiency of the equipment.

\section{5) Real-time monitoring}

The operator can monitor the machine and check the running status of the machine in real time.

\section{6) Fault management}

System can detect the abnormality of the device and notify the operator via SMS or webpage reminder, and the fault information will be added to the fault log.

\section{SYSTEM DESIGN}

\section{A. Overall system design}

The overall design of the system is to carry out the overall plan for the previous analysis, and provide concrete solutions for the realization of the system. The organic waste recycling operation management system not only needs realtime monitoring of the equipment, but also can manage the equipment and improve the working efficiency of the users. According to business function, business process, we design corresponding function and business logic design. And by analyzing the data and entity types involved in the system, the design of the database will be completed.

\section{B. System architecture}

The architecture of the software system is as follows.

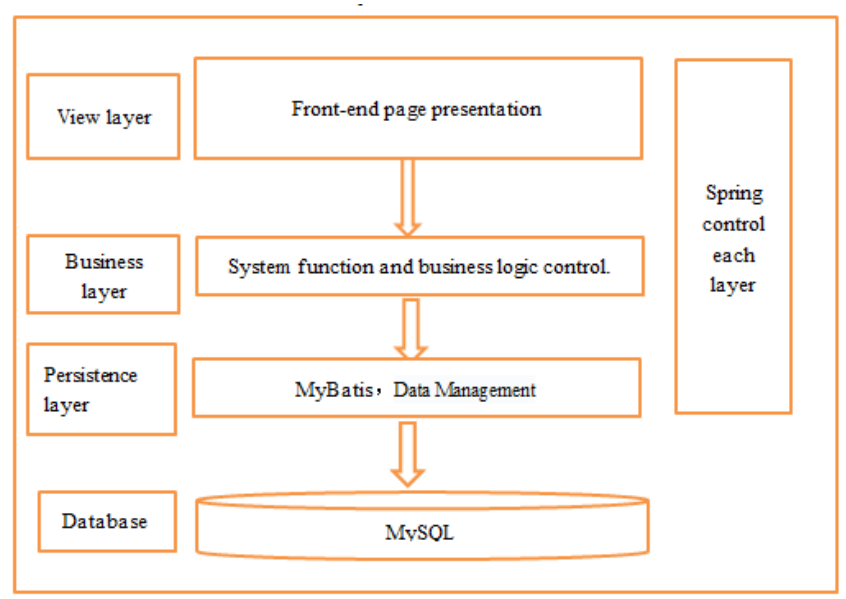

Figure 1. System architecture

View layer handles the interface presentation and user interaction.

Business layer handles the request sent by the user and returns the result of the request to the view layer. At the same time, you will get the data needed by the view layer by calling the persistence layer's mapper interface.

Persistence layer connects to the database, and the data will be processed by the mapper interface.

\section{System function design}

Through the business analysis of the system, the system function is divided into the following contents according to the module.

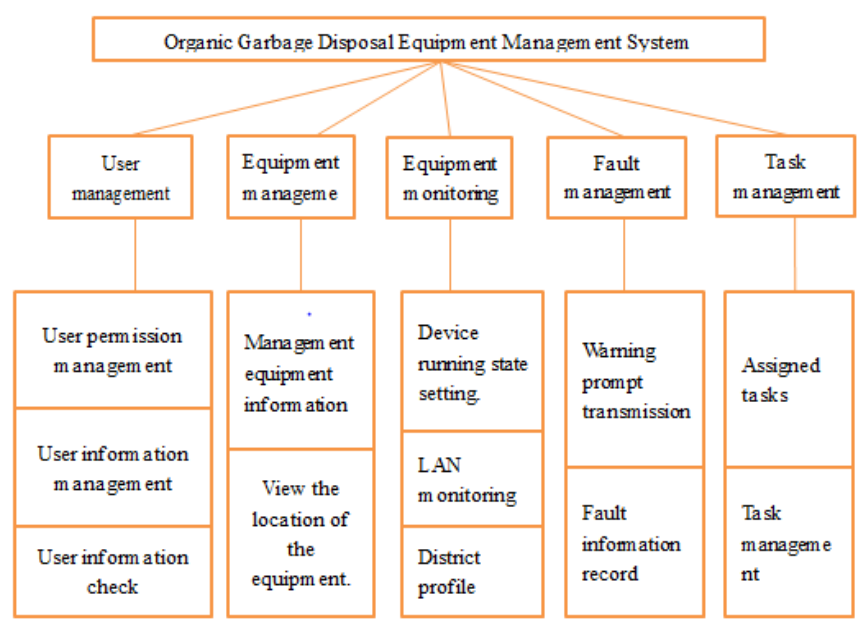

Figure 2. Function module

The functions of each module are as follows:

\section{1) User permission management}

Different users have different executable rights, and from the business logic, it needs to set different roles for different users, which is conducive to the system's safe and stable operation. 


\section{2) User information management}

Manage user information, including add, delete, modify, and other basic operations.

\section{3) Personal information view}

View all personal information and be able to change the password.

\section{4) Equipment information management}

Management equipment information, including adding, modifying, and deleting equipment information and other basic operations.

\section{5) Equipment location view}

Display the corresponding location on the map according to the device address.

\section{6) Device running state setting.}

The user sets the internal humidity, temperature and power of the device during operation, thus making the device in a high efficient state of processing.

\section{7) Single device monitoring}

Simulates the data sent by the sensor and monitors the state of a single device in real time. The state parameters include the internal temperature of the device, the internal humidity of the equipment and the power of the equipment.

\section{8) District profile}

Carry out statistical analysis of equipment quantity information in the area.

\section{9) Warning prompt sending}

When the temperature, humidity or power of the device exceeds the threshold, the system will send a warning to the device owner via text message or the page pop-up box.

\section{0) Fault information record}

When the device fails, the system records the specific fault information. The user needs to modify the status of the fault according to the fault.

\section{1) Assignment of tasks}

The administrator arranges specific tasks through the system and pushes them to the executor.

\section{2) Task management}

The user can update the status of the task in real time according to the task. When the task is completed, it can be submitted to the administrator for review or continued to be pushed to the next executor. Ordinary users manage their own tasks, and administrators can manage all tasks.

\section{SYSTEM IMPLEMENTATION}

\section{A. Holistic structure}

The system is managed by maven, and the required reference packages are placed in the pom.xml.First,we deploy the package structure according to the system function.
The functions of each package are as follows.

- Com.ssm.controller contains the related classes of the Spring controller.

- Com.ssm.entity contains the entity types associated with the project.

- Com.ssm.mapper contains the relevant interface files and corresponding XML files for the operation database.

- Com.ssm.service contains the Service Interface associated with Spring.

- Com.ssm.serviceImpl is the implementation of the interface in com.ssm. service.

- The mybatis package contains the configuration file sqlmapconfig.xml for mybatis.

- Spring packages contains spring-related configuration files, including the database configuration management file applicationcontextdao.xml and view management configuration file springmvc.xml and spring security configuration file applicationcontent-security.xml.

- Db.properties is the connection profile for the database.

- Log4j.properties is the configuration file for the output log.

- Src/main/webapp contains the JSP pages and the imported JavaScript files.

\section{B. Function implementation}

\section{1) Login inplementation}

Input user name and password, the corresponding form will be submitted to the information to the spring security processing, if the information from Spring security that match in the database information, it will be send to the corresponding user requests, load the corresponding menu resources, access to the system home page url. If it does not match to the corresponding information, redirect to the login page. Spring security needs to implement this functionality, in addition to implementing the corresponding configuration in applications-security.xml.

\section{2) Equipment management information}

User can view the information of the device, add, modify, delete, search, and export the required information to excel.

After clicking the location button, the user can view the location of the device. The map uses Baidu Map interface to realize, click the geographical position button and then the location of the device and the device number information will be displayed on the map.

\section{3) Employee management}

User can view the basic information of the employee and add, delete or find there information. You can also set the users' role. And you can select the columns you want to export to an Excel. The Table uses bootstrap Table to dynamically load employee information. 


\section{4) District profile}

Click the district profile button, the map of the country will be displayed, and the depth of the color will be directly proportional to the number of devices. This part is mainly showing the distribution of the equipment from different regions of the country, it uses Echarts to implement, and complete the drill down function of secondary map, click each province will enter the corresponding city's information interface, each city's information using a scatter diagram showing the number of devices. When the mouse is hovering over the provinces, the relevant devices amount will be prompted.

\section{5) LAN monitoring}

User can check the temperature, humidity and power of the device, and the warning threshold, and modify or delete information of the device. Click on the status button to view the real-time state of the device, and the real-time state data is simulated using the random number. The dynamic line chart is realized by Echarts.

The temperature, humidity and power data of the device will be transmitted to controller in real-time. If any abnormal data is found, the device will send SMS messages to the equipment manager. SMS prompts use the SMS interface provided by the Internet provider.

\section{6) Task list}

Users can categorize tasks according to the urgency of the task, and can delete task information and manage the tasks.

\section{7) Fault $\log$}

When a device fails, the system generates a fault log. User can check the fault information of the device and solve the state of the fault.

\section{CONCLUSION}

The organic garbage disposal equipment management system achieves multiple functions such as the rights management, user management, equipment management, equipment monitoring, fault management and early warning, task management, and provides reference for garbage disposal equipment information management.

The system generally operates normally and smoothly. Graphical interactive interface is pleasing to the eye. It can respond quickly to various operations like addition, deletion, search etc.

It can effectively improve the management efficiency of the equipment, optimize the business process, reduce the investment in management, and improve the modern management level of the enterprise.

There are some deficiencies in the system, which need further improvement:

(1) The real-time monitoring part of the equipment uses the simulation data. In practice, it needs to receive the data sent back by the sensor. The integration will be realized in the follow-up work.

(2) When the number of devices is huge, the query speed of the system will be reduced. Optimization of the query algorithm is needed to improve the response speed of the system.

(3) The regional general situation only counts the number of devices in different regions, and it could analyze more factors, such as equipment model, abnormal equipment, etc.

\section{REFERENCES}

[1] Chi Xun,Wang Baojie,Study on Composting Treatment Pattern of Rural Living OrganicWaste,Journal of Shandong Agricultural University. Natural Science,china,vol.48,pp.749-751,2017

[2] Hu Shang-qin,Hu Guo,Research on producing the alcohol from the organic garbage fermentation,1st International Conference on Energy and Environmental Protection (ICEEP 2012),Advanced Materials Research vol. 512,pp. 455-458,2012

[3] ZHANG Xilin,Study on the Garbage Disposal in Urban Park Based on the Circulation Ecoomy Theory,Journal of Anhui Agricultural Sciences vol.35 pp. 817-818,822,2007 